\title{
MEDICINA PALIATIVA: FILOSOFÍA Y CONSIDERACIONES ÉTICAS
}

\section{Robert Twycross, DM FRCP FRCR}

Macmillan Clinical Reader in Palliative Medicine, Oxford University, U.K.

Correspondencia: Dr. Robert Twycross, M.D. The Oxford International Centre for Palliative Care, Churchil Hospital, Oxford, OX 3 7LJ, UK. Fax: (44-1865) 225599 e-mail: rob.twycross@ st-peters.oxford.ac.uk 



\section{MEDICINA PALIATIVA: FILOSOFÍA Y CONSIDERACIONES ÉTICAS*}

Robert Twycross

\section{Resumen}

Después de resumir el origen histórico y el desarrollo que tuvieron los antiguos hospicios, el autor analiza la filosofía que ha inspirado a la Medicina Paliativa desde sus inicios hasta nuestros días en las diferentes partes del mundo. La aparente paradoja que supone encontrar vida y alegría en medio de la muerte y del sufrimiento se explica por el ethos peculiar que orienta el trabajo en equipo, un ethos fundado en la compasión humana práctica. A pesar de enfatizar que la ética de la Medicina Paliativa no es otra que la de la medicina en general, el autor se detiene a analizar algunos de los dilemas éticos más relevantes en el acompañamiento a los pacientes moribundos, como son la comunicación de la verdad, la identificación de las terapias apropiadas, el suministro de nutrición e hidratación y el debate en torno a la eutanasia y el suicidio médicamente asistido. El hilo conductor del trabajo es la necesidad de brindar a los pacientes terminales un acompañamiento profesional interdisciplinario, altamente calificado y personalizado, que tome en cuenta la totalidad de las dimensiones tanto del paciente como de su familia.

PALABRAS-CLAVE: Medicina Paliativa; Muerte y moribundos; Actitudes sociales hacia la muerte; Atención a moribundos; Enfermo terminal; Final de la vida; Eutanasia; Bioética.

Texto traducido del inglés por la Dra. Paulina Taboada, Centro de Bioética, P. Universidad Católica de Chile.

\section{Resumo}

Após resumir a origem histórica e o desenvolvimento que tiveram os antigos hospitais, o autor analisa a filosofia que inspirou a Medicina Paliativa, desde seus primórdios até nossos dias em diferentes partes do mundo. O aparente paradoxo que persiste ao tentar-se encontrar vida e alegria na morte e no sofrimento explica o ethos particular que orienta o trabalho em equipe, um ethos fundado na prática da compaixão humana. Apesar de enfatizar que a ética de Medicina Paliativa não é outra que não a da própria medicina, o autor detem-se na análise de alguns dilemas éticos relevantes no acompanhamento de pacientes moribundos, quais sejam: a comunicação da verdade, a idenficação de tratamentos adequados, o oferecimento de nutrição e hidratação. Levanta, também, o debate em torno da eutanásia e do suicídio assistido. O fio condutor do trabalho prende-se à necessidade de proporcionar aos pacientes terminais um acompanhamento profissional interdisciplinar qualificado e personalizado e que leve em conta a totalidade das dimensões humanas do paciente e de seus familiares. 


\begin{abstract}
After summarizing the historical background and development of former hospices, the author analyses the philosophy that has inspired Palliative Medicine from its selfsame origins up to the present in different countries in the world. The apparent paradox that supposes to find life and joy amidst death and suffering explains the peculiar ethos that guides teamwork, an ethos grounded on practical humane compassion. Despite emphasizing that Palliative Medicine ethics is not different from the one of general medicine, the writer pauses to consider some of the most relevant ethical dilemmas related to accompaniment of dying patients, such as truth disclosure, identification of appropriate treatments, nutrition and hydration supply, and the debate on euthanasia and physician assisted suicide. The connecting theme of the work is the necessity of providing terminal patients with an interdisciplinary professional accompaniment — highly qualified and personalized - intended to consider the totality of patients' and families' human dimensions.
\end{abstract}

KEY-WORDS: Palliative Medicine; Death and dying; Social attitudes towards death; Care for dying; terminally ill; Endof-life; Euthanasia; Bioethics.

\section{Résumé}

Après avoir résumé l'origine historique et le développement des hospices anciens, l'auteur analyse la philosophie qu'inspire la Médicine Palliative, depuis ses origines jusqu'à nos jours et ce dans les différents parties du monde. L'apparent paradoxe que consiste à trouver la vie et la joie au milieu de la mort et de la souffrance, est expliquée par l' ethos particulier que guide le travail en equipe, un ethos fondé sur la compasion humaine pratique. L'auteur, en dépit de souligner que l'éthique de la Médicine Palliative n'est pas différente de celle de la médicine en générale, analyse quelques uns des dilemmes éthiques le plus importants dans l'accompagnement des patients moribons, tels que la communication de la vérité, l'identification des thérapies adéquates, la nutrition et l'hydratation et le débat à propos de l'euthanasie et du suicide médicalement assisté. Le fil conducteur du travail est le besoin de donner aux patients en phase terminale un accompagnement personalisé et multidisciplinaire de la part des professionels hautement qualifié, qui tiennent compte de la totalité des dimensions impliquées, aussi bien du patient que de la famille.

MOTS CLÉS: Médicine Palliative; Mort et Moribons; Attitudes sociales envers la mort; Assistance aux moribonds; Malade terminal; Fin de la vie; Euthanasie; Bioéthique. 


\section{Introducción}

La Medicina Paliativa es la atención activa y total a los pacientes y a sus familias, por parte de un equipo multiprofesional, cuando la enfermedad ya no responde a tratamientos curativos y la expectativa de vida es relativamente corta. La palabra "paliativa" deriva del vocablo latino pallium, que significa manta o cubierta. Así, cuando la causa no puede ser curada, los síntomas son "tapados" o "cubiertos" con tratamientos específicos, como por ejemplo, analgésicos y antieméticos. Sin embargo, la Medicina Paliativa es mucho más amplia y profunda que la mera "sintomatología". El término implica un enfoque holístico que considera no sólo las molestias físicas, sino también las preocupaciones psicológicas, sociales y espirituales. Sus principales objetivos son:

- Aliviar el dolor y los otros síntomas molestos que presentan los pacientes;

- Atender psicológica y espiritualmente a los pacientes para que puedan aceptar su propia muerte y prepararse para ella en la forma más completa posible;

- Ofrecer un sistema de apoyo que ayude a los pacientes a llevar una vida lo más activa y creativa posible hasta que sobrevenga la muerte, promoviendo de este modo su autonomía, su integridad personal y su autoestima;

- Brindar un sistema de apoyo que ayude a las familias a afrontar la enfermedad del paciente y a sobrellevar el período de duelo.

La Medicina Paliativa afirma la vida y reconoce que el morir es un proceso normal. No busca ni acelerar ni posponer la muerte. No está dominada por la "tiranía de la curación", y se opone también firmemente a la eutanasia.

\section{Filosofía de los cuidados paliativos}

La Medicina Paliativa se ha desarrollado, en gran parte, como resultado de la visión e inspiración inicial de Dame Cicely Saunders, fundadora del Hospicio St. Christopher en Londres, en 1967. Hace siglos, "hospicio" significaba lugar de reposo para viajeros o peregrinos. La palabra sobrevivió en conexión con hospitales conventuales o asilos. A Dame Cicely le atrajo este nombre pues quería proveer un tipo de atención que combinara las habilidades de un hospital con la hospitalidad reposada y el calor de un hogar. En un hospicio, el centro de interés se desplaza desde la enfermedad hacia el paciente y su familia; desde el proceso patológico, hacia la persona.

Los pacientes con enfermedades terminales frecuentemente necesitan más atención que aquellos cuya enfermedad es curable. Así, la Medicina Paliativa ofrece un "cuidado terminal intensivo". Requiere de habilidades profesionales de alto nivel y de un cuidado experto, individualizado para cada paciente, atento a los detalles y sensible, que consume mucho tiempo. La Medicina Paliativa es distinta de la geriatría y a la atención de pacientes crónicos, dos especialidades con las que frecuentemente se la compara:

"contiene muchas de las gratificaciones de la cirugía, pues opera en el contexto de intervención de crisis; de la medicina interna, pues invita a la fina titulación de los esquemas terapéuticos para síntomas molestos; de la psiquiatría, pues trata con ansiosos, deprimidos y deudos"

La Medicina Paliativa se desarrolló como una reacción frente a la medicina moderna altamente tecnificada. Halina Bortnowskauna filósofa polaca, escritora y voluntaria en un hospicio- ha contrastado el ethos de la

Esta y las siguientes citas corresponden a una traducción propia (P.T.) de la cita original en inglés (nota de la traductora). 
curación con el ethos de la atención ${ }^{i 1}$. Esta escritora define ethos como "una constelación de valores sostenidos por la gente". El ethos de la curación incluye las virtudes militares del combate: no darse por vencido y perseverancia; contiene necesariamente también algo de dureza. El ethos de la atención, en cambio, tiene como valor central la dignidad humana, enfatizando la solidaridad entre el paciente y los profesionales de la salud, una actitud que resulta en una "compasión efectiva". En el ethos de la curación "el médico es el general", mientras que en el de la atención "el paciente es el soberano". Es importante otorgar al paciente el poder de decidir lo más posible, mientras sea posible.

Así, la Medicina Paliativa busca evitar que los últimos días se conviertan en días perdidos ${ }^{\text {iii }}$, ofreciendo un tipo de atención apropiada a las necesidades del moribundo. A pesar de haber sido descrita como "de baja tecnología y alto contacto", la Medicina Paliativa no se opone intrínsecamente a la tecnología de la medicina moderna, pero busca asegurar que sea el amor y no la ciencia la fuerza que oriente la atención al paciente. Las investigaciones y los tratamientos de alta tecnología sólo se utilizan cuando sus beneficios superan claramente las posibles cargas. La ciencia se utiliza al servicio del amor y no viceversa. La Medicina Paliativa es un intento por restablecer el rol tradicional de médicos y enfermeras: "curar a veces, aliviar frecuentemente, confortar siempre".

ii El autor utiliza aquí un juego de palabras en inglés, que no es posible conservar en la traducción al castellano: “... contrasted the ethos of cure with the ethos of care..." (nota de la traductora).

iii El texto inglés introduce aquí un juego de palabras que no es posible conservar en la traducción al castellano: “...to prevent last days becoming lost days ..." (nota de la traductora).

\section{Orígenes}

\section{a) Hospicios medievales}

El origen de los hospicios se remonta a Fabiola, matrona romana que en el siglo IV abrió su casa para los necesitados, practicando así las "obras de misericordia" cristianas: alimentar a los hambrientos y sedientos, visitar a los enfermos y prisioneros, vestir a los desnudos, acoger a los extraños. En ese tiempo, la palabra "hospicio" significaba tanto anfitrión, como huésped; "hospitium" incluía tanto el lugar donde se daba hospitalidad, como la relación que resultara de ello. Este énfasis es central para la Medicina Paliativa hasta el día de hoy. Más tarde, la Iglesia procuró llevar la carga del cuidado de los pobres y de los enfermos, hecho que continuó hasta la Edad Media. En Gran Bretaña esto tuvo un término abrupto con la disolución de los monasterios en el siglo XVI. Ninguno de los primitivos hospicios se inició específicamente con el fin de atender a los moribundos; sin embargo, ellos invitaban a la gente a quedarse mientras necesitara ayuda, incluyendo así a los moribundos. Después de la disolución muchas personas tienen que haber muerto en su casa en gran pobreza y disconfort.

\section{b) Hospicios para moribundos}

El primer hospicio fundado específicamente para moribundos es probablemente el de Lyon, en 1842. Después de visitar pacientes cancerosos que morían en sus casas, Madame Jeanne Garnier abrió lo que ella llamó un hospicio y un Calvario. Continuó abriendo muchos otros, la mayoría de los cuales aún existen. En Gran Bretaña, el renacimiento de esta palabra ocurrió en 1905, con el hospicio St. Joseph de las Hermanas de la Caridad, en Hackney. Su fundadora, la Madre Mary Aikenhead, había abierto anteriormente un hospicio para moribundos en Dublín en 1879, pero al parecer no hubo ninguna conexión entre ella y Madame Garnier. 
En ese mismo período se abrieron en Londres otros hogares, incluyendo el hospicio St. Columba, en 1885, el Hostal de Dios, en 1892, y el St. Luke's Home para pobres moribundos, en 1893.

El St. Luke's Home fue el único fundado por un médico, el Dr. Howard Barret. De todos estos hogares, el del Dr. Barret era, en principio, el más similar a los "hospicios modernos", lleno de un interés particular y personal por los pacientes. El Dr. Barret dejó una serie de Reportes Anuales, en los que escribe no de "los pobres" o de "los moribundos" en general, sino de personas individuales y de sus desoladas familias, que quedaban en la casa sin apoyo. Posteriormente, en 1911, Douglas Macmillan, un empleado civil, fundó la National Society for Cancer Relief, que concede donaciones económicas a familias de pacientes cancerosos moribundos en sus casas, permitiéndoles acceder a ciertas medidas de confort extra como frazadas, calefactores y comida.

\section{c) Hospicio St. Christopher}

Quizás el siguiente evento con más significado fue el creciente interés de una asistente social en las necesidades de los pacientes moribundos del hospital St. Thomas, en Londres. Su nombre era Cicely Saunders y su interés había sido gatillado en 1948 por su encuentro con David Tasma, un judío refugiado de Polonia que estaba muriendo de cáncer. Juntos habían discutido sobre el tipo de institución que mejor podría satisfacer sus necesidades. Además del alivio del dolor, él quería la "cercanía de alguien que lo considerara como persona”. Este paciente le dejó a Cicely la suma de 500 libras esterlinas en herencia "para ser una ventana en tu hogar".

El camino desde 1948 a 1967 fue largo y duro. Cicely Saunders trabajó por las tardes como enfermera voluntaria en el St. Lukes Home durante 7 años. Mientras estaba allí, aprendió el valor de utilizar opioides fuertes por vía oral en esquema horario — especial- mente morfina y diapomorfina - para aliviar el dolor severo relacionado al cáncer. Para la antigua graduada en inglés de Oxford, fue necesario estudiar ciencias e ingresar a la escuela de Medicina. Después de recibirse, Dr. Saunders se convirtió en la primera doctora de tiempo completo que trabajó en el hospicio St. Joseph, donde introdujo el uso regular de analgésicos orales a horarios en vez del uso de inyecciones a demanda (o PRN). También desarrolló otros aspectos del manejo de síntomas y comenzó a planear su propio hospicio.

El hospicio St. Christopher se abrió en 1967, al sur de Londres, con 54 camas y acomodación adicional para 16 personas ancianas frágiles. Atrajo mucho interés desde el exterior y pronto se convirtió en el punto de encuentro para un movimiento de protesta que anhelaba un mejor trato para los moribundos, rechazados por un sistema de salud que a mediados del siglo XX se había dejado seducir progresivamente por el glamour de las terapias curativas y el resplandor de la alta tecnología.

\section{d) Evolución posterior}

Debido a que la gente con SIDA tiene frecuentemente necesidades físicas y no-físicas diferentes, en muchas ciudades se han establecido servicios de cuidados paliativos separados para ellos. Sin embargo, en la mayoría de los establecimientos la atención de los pacientes con SIDA se realiza junto a los pacientes con cáncer. Un número creciente de hospicios está atendiendo también a pacientes en estadios terminales de enfermedades respiratorias, cardíacas y renales, a pesar de que los números son todavía relativamente pequeños.

Desde mediados de 1980 se han establecido varios hospicios para niños. El primero de ellos fue la Helen House, situada en el terreno de un convento de Oxford. Se construyó como resultado del entusiasmo y compromiso de la Hermana Francisca Domini- 
ca, entonces Madre Superiora de la Orden. Los hospicios de niños son muy distintos a los de adultos. Generalmente tienen sólo ocho camas y mayores facilidades para los miembros de la familia. Muchos de los niños tienen enfermedades neurológicas degenerativas, frecuentemente asociadas a alteraciones metabólicas congénitas. La mayoría de las internaciones están destinadas a conceder un descanso a la familia y se repiten a intervalos regulares cada 2 a 3 meses, durante algunos o incluso muchos años. El número de defunciones por año es relativamente pequeño. En contraste con los adultos, los niños con leucemia o con cáncer que no se curan son mantenidos en los servicios especializados (pediatría) de los grandes hospitales, ofreciendo una atención extendida a la casa cuando se desea y siempre que sea posible.

La Asociación de Medicina Paliativa de Gran Bretaña e Irlanda se formó en 1985 y dos años más tarde el Reino Unido se convirtió en el primer país en reconocer la Medicina Paliativa como especialidad médica. Todas las escuelas de enfermería y medicina del Reino Unido incluyen los cuidados paliativos como parte de su currículo. También se han establecido programas de postgrado en cuidados paliativos, que incluyen varios diplomados y cursos conducentes a grados académicos.

\section{Desarrollo internacional}

\section{a) Estados Unidos de Norteamérica}

La influencia de Cicely Saunders se extendió progresivamente a otros países y su "llama" fue tomada y llevada a la práctica por un ejército de entusiastas de segunda y tercera generación. Inevitable, pero acertadamente, lo que se ha desarrollado ha sido con frecuencia sustancialmente distinto del modelo británico. En Estados Unidos, a pesar del desarrollo temprano de un servicio de internación en el hospicio de Connecticut, la mayoría de los hospicios proveen solamente apoyo para la atención domiciliaria. Además, mientras que el director médico de un hospicio en Inglaterra es generalmente un médico de jornada completa (o casi completa), en Estados Unidos el director médico habitualmente tiene una jornada parcial y algunas veces meramente nominal. De hecho, el ethos del movimiento hospice en Estados Unidos fue inicialmente y durante muchos años prevalentemente anti-médico. A fines de los '70 y comienzos de los ' 80 , el movimiento hospice en los Estados Unidos era una organización popular, comunitaria, dirigida por voluntarios y enfermeras, que con frecuencia desincentivaban o limitaban la participación de los médicos.

Esta reacción inicial se debió parcialmente a que las enfermeras fueron más rápidas en captar la visión de los hospicios y también en parte a que el cuerpo médico adoptó generalmente una postura contraria. En los Estados Unidos, la atención de salud se basa mayoritariamente en empresas aseguradoras, existiendo un considerable desincentivo económico tanto para los médicos como para los hospitales, ya que inicialmente la atención en hospicios no era cubierta por los seguros. Esto significaba que ofrecer cuidados paliativos a un paciente suponía una pérdida de ingresos para los médicos y para los hospitales cuando el paciente necesitaba ser internado. A pesar de que en la actualidad los cuidados paliativos son por ley un componente de los programas de las aseguradoras de salud, las antiguas actitudes y prejuicios aún persisten. Parte de la reticencia de los médicos americanos para aceptar la Medicina Paliativa parece venir de la reticencia que tiene la American Medical Asociation a reconocer los logros realizados por el movimiento hospice en Estados Unidos durante los últimos 20 años. En la actualidad, cerca de 2000 hospicios están afiliados a la American National Hospice Organization. 


\section{b) Polonia}

En Polonia, en cambio, la Medicina Paliativa floreció a comienzos de 1980 con la prohibición de la unión obrera Solidaridad por parte del gobierno comunista de esa época. Los doctores de las academias médicas que se hacían miembros de Solidaridad eran removidos de sus cargos y obligados a trabajar en hospitales estatales de menor prestigio. En Gdansk, cuna de Solidaridad, muchos de estos doctores decidieron desarrollar un programa de hospicios bajo la protección de la Iglesia Católica, como una forma de manifestar su oposición al gobierno.

En éstas y en otras organizaciones voluntarias similares los médicos desempeñaban la mayoría de los roles. De este modo, los médicos paliativistas polacos eran originalmente algo así como un combinación entre un médico general sustituto y una enfermera especializada en cuidados paliativos. En la actualidad existen en Polonia muchos grupos de paliativos y algunos servicios de internación hospitalaria. Sin embargo, para los médicos se trata aún de un servicio voluntario en la mayoría de los casos, debiendo ganar su sustento mediante su especialidad básica y trabajando horas extraordinarias en los hospicios.

\section{c) Una red en expansión}

En otros países, el desarrollo de la Medicina Paliativa ha reflejado también las necesidades, oportunidades y recursos locales. En algunos países, la Medicina Paliativa es, en mayor o menor grado, una copia del modelo británico (p. ej. Canadá, Australia, Nueva Zelandia); en otros, refleja más el modelo americano y polaco. En todas partes se ha encontrado con la resistencia inicial de los oncólogos y con la indiferencia de los estamentos médicos. Las razones para ello son debatibles, pero el contraste entre el ethos de la curación y el ethos de la atención planteado por Halina Bortnowska probablemente ofrece la clave para encon- trar la respuesta. Muchos países han fundado asociaciones nacionales multiprofesionales de Medicina Paliativa. Existe, además, una asociación nórdica y otra europea de Medicina Paliativa.

\section{d) La Organización Mundial de la Salud}

Desde mediados de los 80, otro lobby poderoso para la Medicina Paliativa ha sido la Organización Mundial de la Salud (OMS). Bajo el visionario liderazgo del Dr. Jan Stjernsward, la Unidad de Cáncer de la OMS ha llevado a cabo una campaña estimulando a los países a desarrollar programas exhaustivos de control del cáncer, que incluyen:

- Prevención

- Detección precoz y tratamiento curativo

- Alivio del dolor y Cuidados Paliativos.

La publicación de Alivio del Dolor en Cáncer, en 1986 (Organización Mundial de la Salud, 1986), ha sido un catalizador importante en este sentido. Este libro, que incorpora el método de la OMS para el alivio del dolor relacionado con cáncer, es la segunda publicación más traducida de la OMS. Además del inglés, está disponible en 19 otras lenguas: árabe, brasilero, búlgaro, chino, croata, checo, francés, alemán, guajiri, hindú, húngaro, italiano, japonés, portugués, ruso, español, tailandés, turco y vietnamés. Esta publicación, con más de un cuarto de millón de copias vendidas y distribuidas, refleja una creciente conciencia del problema del dolor en cáncer. El método de la OMS enfatiza que un pequeño número de drogas relativamente baratas, incluyendo la morfina, son los principales apoyos para el manejo del dolor en cáncer. Pruebas en terreno han demostrado la eficacia de estas orientaciones en la mayoría de los pacientes con cáncer. El método original de la OMS ha sido actualizado y publicado en la segunda edición de Alivio del Dolor en Cáncer. 


\section{Estrategia general}

En la práctica, la mayoría de los pacientes tratados en hospicios o por equipos de cuidados paliativos tiene cáncer. La Medicina Paliativa es parte de un enfoque global para el control del cáncer. El tratamiento curativo mediante cirugía, radioterapia y/o quimioterapia frecuentemente tiene que ser agresivo ("radical") si se quiere que sea exitoso. Este tipo de tratamiento se justifica solamente:

- Cuando la curación es posible;

- Cuando hay una posibilidad realista de prolongar la sobrevida;

- En ensayos clínicos sobre un método de tratamiento nuevo, potencialmente efectivo.

La decisión de limitar la terapia curativa y ofrecer cuidados paliativos es una decisión crucial; los intentos por curar lo incurable no van en el mejor interés del paciente que realmente necesita cuidados paliativos.

Incluso, el tratamiento anti-canceroso no radical (llamado tratamiento paliativo) no debería ofrecerse a menos que exista una posibilidad real de que las cargas físicas, psicológicas y financieras del tratamiento sean sobrepasadas por los potenciales beneficios que ella ofrece al paciente. Desde el punto de vista del paciente, prolongar la vida por unas semanas o meses, generalmente no es una justificación adecuada para iniciar una terapia cuyo costo será una calidad de vida marcadamente reducida durante la mayor parte del tiempo extra, a causa de los efectos adversos o de la ruina económica inducida por los gastos incurridos. En la mayoría de los países desarrollados, a pesar de que la sobrevida general del cáncer a cinco años plazo excede el $50 \%$, cerca del $70 \%$ de todos los pacientes con cáncer aún necesita, tarde o temprano, cuidados paliativos. En países en vías de desarrollo la cifra alcanza el 90\%. En la práctica, hay algunos pacien- tes que se benefician de tratamientos paliativos y de cuidados paliativos simultáneamente. En estos casos, lo adecuado es ofrecer un paquete de cuidados compartidos, involucrando tanto al equipo de oncología como al de Medicina Paliativa.

\section{Una paradoja}

Aquellos que visitan un hospicio por primera vez frecuentemente comentan que no es el lugar oscuro y deprimente que habían imaginado. Encuentran, en cambio, un lugar que irradia vida e incluso alegría. Es un descubrimiento que resulta extraño: vida y alegría en medio de la muerte y la angustia. Sin embargo, es quizás precisamente en esta paradoja donde reside el "secreto" de la Medicina Paliativa.

Procurar crear (artificialmente) una paradoja de esta naturaleza es probablemente imposible. Irradiar vida y alegría en medio de la muerte y de la angustia es el resultado final de realizar actividades muy a ras de tierra, que tiene su origen en una compasión humana práctica, como son los cuidados de enfermería, el manejo de los síntomas y el apoyo psicológico. Esto es, a su vez, producto de una actitud de respeto por el paciente y de una actividad corporativa en la que el individualismo es balanceado por el trabajo en equipo y viceversa. La "casa del hospicio" (Fig. 1) es una buena imagen para expresar esto, con sus piedras angulares de aceptación ("pase lo que pase no te abandonaré") y de afirmación ("puedes estar muriendo, pero tú eres importante para nosotros"). El cemento que mantiene unidos los distintos componentes de la casa está dado por la esperanza, la apertura y la honestidad.

Cuidar de los moribundos no es fácil. Un médico lo describió como "extremadamente doloroso pero muy recompensante". Es difícil pero, paradójicamente, con frecuencia tiene una nota positiva, no sólo para los profesionales de la salud. Después de la muerte de su mujer, un periodista escribió lo siguiente: 
"Por supuesto que un cáncer terminal es inefablemente horrible. Este aspecto no necesita ser enfatizado. Sin embargo, la bendición que es corolario de ese horror resulta más difícil de imaginar... Yo creo que mi esposa supo más de nuestro amor y nosotros más del suyo durante esos meses espantosos, que en cualquier otro tiempo... El sufrimiento de una enfermedad larga y terminal no es un desperdicio. Nada que pueda crear esa ternura puede ser un desperdicio. Como destructor, al cáncer no le gana nadie. Pero también es un sanador; un agente de sanación".

Un paciente moribundo con una enfermedad de motoneurona (parálisis) llamaba a la enfermedad terminal "enfermedad del encuentro". Después de varias semanas hospitalizado en el hospicio St. Cristopher dijo: "Lo he visto una y otra vez: paciente y familia; paciente y paciente; paciente y equipo médico... enfermedad del encuentro".

\section{Ética de la medicina paliativa}

La ética de la Medicina Paliativa es, por supuesto, la misma que la de la medicina en general. Quizás el mayor desafío que enfrentan los médicos en relación a la Medicina Paliativa es la cuestión acerca de la veracidad con los pacientes terminalmente enfermos. La reticencia a compartir con los pacientes la verdad acerca de su condición se origina, en última instancia, en el miedo que tiene el propio médico a la muerte y en las presiones culturales asociadas. Aún se afirma con frecuencia que decirle al paciente que está terminalmente enfermo destruirá su esperanza y lo llevará irreversiblemente a la desesperación y a la depresión. En realidad, con frecuencia ocurre lo contrario: son la mentira y la evasión lo que aísla a los pacientes tras un muro de palabras o de silencio, que les impide acceder al efecto terapéutico que tiene el compartir sus miedos, sus angustias y sus otras preocupaciones. No es posible practicar la Medicina Paliativa sin un compromiso previo de apertura y honestidad. Por tanto, para muchos médicos el primer desafío ético es el equiparse a sí mismos de buenas habilidades de comunicación $\mathrm{y}$ de sensibilidad. Afortunadamente, la conciencia de esta necesidad ha ido creciendo, pero la carencia global de entrenamiento en este campo es algo aún muy difundido.

\section{a) Tratamiento apropiado}

Los médicos tienen una responsabilidad dual, a saber: preservar la vida y aliviar el sufrimiento. Sin embargo, hacia el final de la vida el alivio del sufrimiento adquiere una importancia mayor en la medida en que la preservación de la vida se hace progresivamente imposible. Parte del arte de la medicina es decidir cuándo el soporte vital es esencialmente inútil y, por tanto, cuándo se debe permitir que sobrevenga la muerte sin mayores impedimentos. Un médico no está obligado ni legal ni éticamente a preservar la vida "a toda costa". La vida debe ser sostenida cuando desde el punto de vista biológico es sostenible. Las prioridades cambian cuando un paciente está claramente muriendo; no hay obligación de emplear tratamientos cuando su utilización puede ser descrita - en el mejor de los casos- como una prolongación del proceso de morir. Un médico no tiene ni el deber ni el derecho de prescribir la prolongación de la muerte.

De este modo, la pregunta no es si "tratar o no tratar", sino "cuál es el tratamiento más apropiado", de acuerdo a las perspectivas biológicas del paciente y a sus circunstancias personales y sociales. El tratamiento que es apropiado para un paciente agudamente enfermo puede no ser apropiado para un moribundo (Fig. 2 y 3). Las sondas nasogástricas, las infusiones intravenosas, los antibióticos, la resucitación cardiopulmonar y la respiración artificial son medidas de soporte que deben ser utilizadas en enfermedades agudas o agudas sobre crónicas para asistir al paciente a través de la crisis inicial hacia la 
recuperación de la salud. Usar esas medidas en pacientes que están próximos a la muerte y sin expectativas de recuperar la salud es habitualmente inapropiado (y, por tanto, mala medicina).

La atención médica es un continuo que va desde la curación completa, por un lado, hasta el alivio sintomático, por el otro. Muchos tipos de tratamientos abarcan el espectro completo, como es el caso de la radioterapia y, en menor medida, de la quimioterapia y de la cirugía. Por tanto, es importante tener claramente presente el objetivo de la terapia al emplear cualquier forma de tratamiento. Los puntos clave que se deben tener en cuenta para decidir qué es apropiado, son:

- Las perspectivas biológicas del paciente;

- El propósito terapéutico y los beneficios de cada tratamiento;

- Los efectos adversos del tratamiento;

- La necesidad de no prescribir una prolongación de la muerte.

A pesar de que la posibilidad de una mejoría inesperada o una recuperación no deben ser ignoradas, hay muchas ocasiones en las que es apropiado "darle una oportunidad a la muerte". No obstante, muchos médicos encuentran extremadamente difícil cambiar de un modus operandi orientado a la curación a uno centrado en las medidas de confort. Quizás sea ésta una de las razones por las que la "voluntad antecedente" está siendo una práctica cada vez más popular en muchos países.

\section{b) Oposición a la eutanasia}

La oposición casi unánime de los médicos y enfermeras paliativistas al suicidio médicamente asistido y a la eutanasia, nace de la observación de la realidad de innumerables pacientes moribundos. En vez de atenuar las actitudes hacia esas opciones, esta observación parece endurecer el antagonismo. Las razones para ello son muchas e in- cluyen tanto la vulnerabilidad y ambivalencia de los pacientes, como el hecho de que la mayoría de los pacientes que solicitan suicidio asistido o eutanasia cambian de opinión cuando se les proveen cuidados paliativos de buena calidad. Otra razón es la aparente imposibilidad de contener esta práctica dentro de ciertos límites si llegara a legalizarse.

El término "eutanasia indirecta" ha sido usado para describir la administración de morfina a pacientes cancerosos con dolor. Esto es incorrecto; dar una droga para disminuir el dolor no puede ser equiparado a dar deliberadamente una sobredosis de ella para terminar con una vida. Acortar marginalmente la vida por el uso de morfina o drogas relacionadas constituye un riesgo aceptable en estas circunstancias (principio del doble efecto). Sin embargo, cuando son usadas correctamente estas drogas son mucho más seguras de lo que comúnmente se supone. Por otro lado, existe evidencia circunstancial de que aquellos pacientes cuyo dolor ha sido aliviado viven más que aquellos cuya nutrición y reposo siguen siendo interferidos por un dolor persistente.

\section{c) Nutrición e hidratación}

Cuando el paciente se aproxima a la muerte, la ingesta de comida y fluidos generalmente disminuye. Es en este punto cuando surge la pregunta acerca de la administración de fluidos por medios artificiales. La opinión tradicional de los hospicios es que cuando el interés por la comida y los fluidos se hace mínimo, un paciente terminal no debería ser forzado a recibirlos. De hecho, comer y beber puede no ser relevante para un paciente en esas circunstancias, en que la atención está volcada hacia el interior o hacia el "más allá". Sin embargo, muchos pacientes moribundos reciben automáticamente fluidos endovenosos cuando no son capaces de mantener un balance hídrico normal. La principal razón de ello es que parece existir la creencia que la deshidratación causaría angustia en una persona cercana a la muer- 
te. Sin embargo, el personal de los hospicios generalmente opina que la deshidratación puede ser beneficiosa, mientras que la hidratación puede ser incluso perjudicial. Se afirma, así, que con la deshidratación el volumen urinario disminuye, existiendo menos necesidad de orinar o cateterizar la vía urinaria y menos episodios de "cama mojada". Se dice que la reducción de las secreciones pulmonares disminuiría la tos, las sensaciones de asfixia y ahogo y la necesidad de aspiraciones de la vía aérea. Asimismo, la disminución de las secreciones gastrointestinales resulta en menos episodios de vómitos en pacientes con obstrucción intestinal. El dolor también puede disminuir a causa de una reducción del edema que rodea las masas tumorales.

La hidratación endovenosa también tiene efectos psicosociales negativos, pues la infusión actúa como una barrera entre el paciente y su familia. Es más difícil abrazar al cónyuge que está conectado a un tubo plástico, y los médicos y enfermeras tienden a distraerse de los aspectos más humanos de la atención por controlar el balance de fluidos y la química sanguínea. Con frecuencia, la familia se angustia cuando los pacientes próximos a la muerte disminuyen drásticamente su ingesta oral. Hay que establecer, entonces, cuáles son sus sentimientos hacia la terapia endovenosa y el significado que le atribuyen, para explicarles la racionalidad de discontinuar esa terapia. La decisión acerca de la conveniencia de rehidratar debe centrarse más en el confort del paciente que en el objetivo de proveer una óptima nutrición e hidratación.

Por otro lado hay quienes afirman que la administración de fluidos por vía hipodérmica (500-1500 ml/24hrs.) evita muchas de las objeciones que se hacen a los otros métodos de hidratación. Sin embargo, hasta la fecha no hay evidencia de que la rehidratación logre que los pacientes estén generalmente más confortables. Se ha reportado que los fluidos hipodérmicos podrían aliviar el delirio en algunos pacientes moribundos. Por otro lado, también se ha reportado una me- joría cognitiva después de retirar la hidratación.

Entre las indicaciones para rehidratar estaría que el paciente sienta sequedad de boca a pesar de un buen cuidado oral. La sequedad oral es común en los pacientes con cáncer y no se relaciona sólo con la deshidratación, sino también con otras causas como las drogas, las infecciones orales, la radioterapia local, la oxigenoterapia y la respiración bucal. Por tanto, es improbable que la sola hidratación artificial resuelva el síntoma de sequedad bucal en la mayoría de los pacientes. Por otro lado, la sequedad de boca puede ser aliviada en gran medida por una higiene oral cuidadosa y con la administración de 1 - $2 \mathrm{ml}$. de agua mediante una pipeta o jeringa en la cavidad oral cada 30 a 60 minutos.

\section{Rehabilitación}

A pesar de que para los extraños resulte probablemente sorprendente, la rehabilitación es una parte integral de la Medicina Paliativa. Muchos de los pacientes terminales están restringidos innecesariamente, con frecuencia por parte de sus mismos familiares, incluso aunque sean capaces de un mayor grado de actividad e independencia. El potencial máximo de un paciente sólo se alcanzará si se alivian los síntomas molestos y si un médico dedicado lo anima amablemente.

No todos los médicos aprecian las oportunidades de rehabilitación que existen en pacientes con cáncer avanzado. Esto se debe, en parte, a la inexperiencia que ellos tienen en cuidados paliativos, pero posiblemente también a las actitudes excesivamente negativas que aún existen en relación al cáncer. Se puede vivir 5 o más años con metástasis óseas de un cáncer de mama. La hormonoterapia puede cambiar radicalmente el pronóstico de un paciente con cáncer de próstata diseminado recientemente diagnosticado. La sobrevida media después del diagnóstico de un cáncer de páncreas puede ser menor a 6 meses, pero eso significa que la mitad de los pacientes vivirá más que eso y 
unos pocos incluso mucho más. Además, el solo alivio de los síntomas puede producir una considerable mejoría a corto plazo.

Como ya se ha dicho, en muchas unidades de cuidados paliativos alrededor del $50 \%$ de los pacientes admitidos por primera vez son dados de alta hacia su casa con sus familiares. Ello muestra que el objetivo de los cuidados paliativos de proveer un sistema de soporte que ayude a los pacientes a vivir lo más activa y creativamente posible hasta la muerte, no es un sentimiento vacío. El concepto de que se puede vivir con cáncer hasta que sobrevenga la muerte es aún extraño para muchos de los pacientes y sus familiares, pero también lo es para muchos médicos y enfermeras. El cáncer es un espectro de alteraciones e incluso cuando es curable las tasas de progresión pueden variar.

No obstante, no todas las unidades de cuidados paliativos adoptan un enfoque rehabilitador en el cuidado de los pacientes. Algunas asumen un enfoque sobreprotector, tendiendo a mantener hospitalizaciones más prolongadas y menos altas. En otras, en cambio, se fomenta la independencia de los pacientes.

En el mejor de los casos, la Medicina Paliativa estimula a los pacientes a ser creativos, restaurando o mejorando así su autoestima. El énfasis en "hacer" en vez de "ser atendido" ayuda a los pacientes a vivir y a morir como personas. En muchos casos, un estímulo amable e imaginativo es todo lo que se necesita para que un paciente inicie una actividad que lo lleve a un sentimiento de mayor bienestar. Consecuente con ello, algunas unidades de cuidados paliativos estimulan la creatividad a través de la escritura de poemas y del arte en general.

\section{Esperanza}

Crear metas realistas con los pacientes es una forma de restablecer y mantener su esperanza. En un estudio se vio que los médi- cos y enfermeras que trabajan en Medicina Paliativa proponían significativamente más metas a sus pacientes que sus contrapartes en un hospital general. Pero la esperanza también está relacionada con otros aspectos de la vida y de las relaciones personales (Tabla 1). La comunicación de verdades dolorosas no tiene por qué ser equivalente a destruir la esperanza; la esperanza de la recuperación puede ser reemplazada por una esperanza alternativa. En los pacientes próximos a la muerte la esperanza tiende a centrarse en:

- En el ser más que en el lograr;

- Las relaciones con otros;

- La relación con Dios o con un "ser superior".

Es posible, por tanto, que la esperanza aumente cuando una persona se aproxima a la muerte, siempre que se mantengan una atención y un confort satisfactorios. Cuando queda poco que esperar, aún se puede esperar no ser dejado solo a la hora de morir, y morir en paz.

\section{Acompañamiento calificado}

Algunos han expresado temor de que la Medicina Paliativa se convierta en una "técnica" más dentro de la gran corriente de medicina altamente tecnificada; una técnica tras la cual los profesionales puedan esconderse y a través de la que puedan ejercer "poder". Este peligro se puede evitar si la Medicina Paliativa continúa siendo una forma de acompañamiento: el acompañamiento de profesionales calificados a aquellos que están muriendo.

En las crisis todos necesitamos compañía. Al morir necesitamos una compañía que pueda explicarnos por qué tenemos dolor, dificultad para respirar, constipación, debilidad, etc.; alguien que pueda explicarnos en términos simples lo que está sucediendo. La 
explicación es clave en la terapia, pues reduce psicológicamente la magnitud de la enfermedad y de los síntomas. La situación deja de estar rodeada de un total misterio cuando hay alguien que puede explicar lo que está pasando. Eso es tranquilizador.

La gente suele comentar a las personas que trabajan en cuidados paliativos: "Supongo que ustedes se endurecen con el tiempo y desarrollan un muro protector". La respuesta es un enfático: “¡No!” Obviamente, el médico y la enfermera paliativistas están más familiarizados con los muchos y variados desafíos que impone una enfermedad terminal y adquieren así una cierta confianza. Sin embargo, eso no es lo mismo que endurecerse. En muchos casos la verdad es justamente lo contrario: a medida que pasan los años se sienten cada vez más vulnerables. La Medicina Paliativa es, y siempre será, extremadamente demandante de los recursos emocionales de los prestadores de salud. Es difícil decirle a un paciente: "Sí, es cáncer" o "Sí, parece que la enfermedad está ganando". Y especialmente difícil cuando el paciente tiene 16 ó 26 años. Incluso a los 76 u 86, no es fácil. De hecho si alguna vez parece fácil el médico puede estar seguro de que ya no está siendo de mucha utilidad para sus pacientes.

Cómo una persona puede brindar apoyo a otra es en última instancia un misterio. La investigación en el campo del consejo ha mostrado que para que una relación terapéutica sea lo máximo de beneficiosa es necesario que el terapeuta manifieste empatía, calidez y autenticidad. Debe enfatizarse, además, el valor del contacto físico. En Anatomía de una enfermedad el autor escribe sobre:

"el total vacío creado por la añoranza inerradicable, persistente y recurrente de un contacto humano cálido. Una sonrisa cariñosa y un apretón de manos tenían más valor incluso que las ofertas de la ciencia moderna, pero estas últimas eran mucho más asequibles que lo primero".
Una enfermera observó que hay dos tipos de médicos: "Médicos al lado de la cama, que se interesan por el paciente y médicos a los pies de la cama, que se interesan por la condición del paciente". Desafortunadamente, muchos médicos usan "comportamientos distanciadores" específicos cuando tratan con sus pacientes. Los médicos necesitarían modificar su comportamiento si quieren ser un verdadero apoyo para los pacientes moribundos y sus familias. Es necesario aprender a "escuchar activamente" para facilitar la expresión de las emociones negativas y de los temores. Una forma de abrir la puerta a las preocupaciones no expresadas del paciente es plantear preguntas abiertas, como por ejemplo: “¿cómo está hoy?”, “cómo ha estado desde la última vez que nos vimos?", "¿cómo ha reaccionado su familia ante la enfermedad?".

\section{Hacia una atención totalmente personalizada}

El alivio del dolor y de los otros síntomas angustiosos es considerado, con razón, el fin primario de la Medicina Paliativa. Allí donde se ha introducido la Medicina Paliativa, la expertise en el manejo de los síntomas ha significado que los pacientes puedan esperar estar prácticamente libres de dolor. También se puede esperar un alto grado de alivio de muchos otros síntomas. Sin embargo, al no estar distraídos y agotados por un dolor no aliviado, los pacientes pueden experimentar mayor angustia emocional y espiritual cuando contemplan la proximidad de su muerte. Son pocos los que hacen esto con equilibrio; la mayoría se defiende a sí mismos psicológicamente de diversas formas; y algunos se ven sobrepasados por la angustia, la rabia o el miedo a lo que les está pasando. En consecuencia, se ha sugerido que un hospicio es un "lugar seguro para sufrir". Los pacientes deben saber que la confusión y la angustia son un signo de que están experimentando una reacción de ajuste mayor 
y no de que se están volviendo locos, ya que el miedo a la locura con frecuencia supera al miedo a la muerte.

Es necesario ofrecer una atención totalmente personalizada (Tabla 3). El equipo debe procurar ayudar a que el paciente dé lo mejor de sí, según su personalidad, su familia, su cultura, sus creencias, su edad, su enfermedad, sus síntomas, sus ansiedades y sus temores. Es necesaria la flexibilidad; hay que saber encontrar a los pacientes allí donde se encuentran social, cultural, psicológica, espiritual y físicamente. No existe algo así como el paciente moribundo típico.

\section{Aspectos espirituales del cuidado}

Históricamente, los hospicios modernos tienen su raíz en la fe cristiana, aunque en la práctica debería decirse más ampliamente: en el teísmo. El compromiso de los judíos ha sido evidente desde los primeros momentos y ahora existe también una asociación de hospicios budista. El trabajo con los moribundos exige fe en la vida. Esto es verdad tanto si ella se expresa como si no. Se estima que la vida tiene un sentido y un propósito aun durante una enfermedad terminal. Esta convicción se manifiesta más en las actitudes y acciones que en las palabras; más en el modo de responder a los moribundos y cuidar de ellos, que en lo que decimos.

Se ha escrito mucho sobre las necesidades emocionales de los moribundos; pero mucho menos sobre los aspectos espirituales de su atención. Sin embargo, la vida humana no está gobernada simplemente por instintos y hormonas. Los deseos humanos se extienden más allá de las necesidades básicas de comida, confort y compañía. La gente se cuestiona; y las criaturas que se cuestionan, preguntan: “¿por qué?” Como dice Nietzsche, "aquel que tiene por qué vivir puede soportar casi cualquier cómo". Cuando está muriendo, mucha gente se cuestiona su vida por primera vez:
“He vivido una buena vida?”

"Nunca le he hecho daño a nadie"

“¿Por qué me ocurre esto a mí?”

¿Qué he hecho para merecer esto?”

Sólo una pequeña minoría conversa sobre estos temas con su médico. Sin embargo, la mayoría lo hace con una enfermera, una asistente social o con parientes o amigos cercanos. La gente es muy perceptiva y es poco probable que los pacientes involucren al personal de salud si sienten que la comunicación a este nivel puede causarles disconfort.

Lo "espiritual" abarca la esencia de lo que significa ser humano; incluye aquellas experiencias de la vida humana que trascienden los fenómenos sensibles. Con frecuencia estas experiencias dan origen a reflexiones teológicas, respuestas religiosas y creencias éticas. Muchas de las características de la "casa de los hospicios" (Fig.1) se relacionan con la dimensión espiritual del hombre: aceptación, afirmación, belleza, creatividad. Aquellos que se acercan al final de su vida a menudo tienen necesidad de perdón y de reconciliación; de restablecer "buenas relaciones".

\section{Trabajo en equipo}

El trabajo en equipo es un componente esencial de la Medicina Paliativa. Trabajo en equipo significa práctica integrada. Sin embargo, sería ingenuo juntar un grupo de personas altamente diversas y esperar que por el solo hecho de llamarlo equipo funcionara como tal. Así como existe la ciencia del manejo de los síntomas y el apoyo psicosocial, así existe también una ciencia del trabajo en equipo. El trabajo en equipo implica coordinación de esfuerzos; facilita la identificación de los recursos disponibles y evita la duplicación estéril. En Medicina Paliativa, el núcleo del equipo generalmente está dado por una enfermera, un médico, una asistente so- 
cial y un clérigo. A pesar de que cada profesión tiene una contribución específica que hacer, inevitablemente existen áreas de sobreposición. Esto es especialmente válido con la enfermería y la medicina. La "difuminación de los roles" es una característica inevitable del trabajo en equipo; también lo es el conflicto y la necesidad de manejarlo en forma constructiva y creativa.

Tener una meta común unifica. Pero aunque pueda ser fácil coincidir en el objetivo general de alcanzar la mejor calidad de vida posible para la persona moribunda, el consenso acerca de lo que constituye una buena calidad de vida es más difícil de alcanzar. No obstante, una ventaja del trabajo en equipo es que la situación del paciente puede ser percibida de un modo más global que lo que generalmente ocurre en el caso de profesionales aislados.

Cuando el personal de un hospicio funciona bien como equipo, juntando sus habilidades y recursos - cuidando unos de otros, así como también de los pacientes y sus familias - parece existir un sentimiento de rectitud; una especie de alegría y de plenitud que muy raramente se encuentran en los lugares de trabajo hoy en día.

\section{Carisma versus rutinización}

Se ha sugerido que cualquier sociedad alterna entre la rutinización a que la lleva la burocracia y el carisma que irrumpe como un fenómeno recurrente. En este contexto, carisma se refiere a la habilidad de individuos excepcionales de actuar como catalizadores de cambio social; es el reconocimiento del impacto que puede tener la personalidad en la producción de innovaciones radicales en las instituciones y en las creen- cias establecidas. En relación a la evolución de la Medicina Paliativa en el Reino Unido durante los últimos 25 años, Dame Cicely Saunders fue claramente una influencia carismática inicial, que fue replicada subsecuentemente por liderazgos similares en otras localidades:

El liderazgo inherentemente carismático e inestable, es lo que no tiene una "maquinaria" organizada a su disposición y cuyo poder no ha sido ganado a través de procedimientos institucionales... Esencialmente creativo e intromisor, promueve una nueva orientación valórica, que choca inevitablemente con la existente.... Según Weber, el liderazgo puede permanecer carismático solamente mientras el número de seguidores es pequeño, es decir, al comienzo de un movimiento exitoso. Cuando se expone a la vida diaria, los movimientos carismáticos se ven confrontados inevitablemente con la necesidad de crear una maquinaria administrativa, con la adquisición de fondos y con el problema de la sucesión, y así comienza el proceso de rutinización.

¿Estará pasando la Medicina Paliativa desde la influencia creativa e innovadora del carisma al cómodo ambiente de la rutinización? Ojalá que no. La mayoría de los programas de Medicina Paliativa aún no han alcanzado la meta de una atención totalmente personalizada. Para que la Medicina Paliativa continúe desarrollándose es necesario mantener una tensión creativa entre el carisma y la rutinización. De otro modo, el movimiento de los hospicios de finales del siglo XX acabará degenerando en un tranquilo monumento que descansa precariamente sobre los laureles marchitos, como ha ocurrido con tantos otros antes que él. 


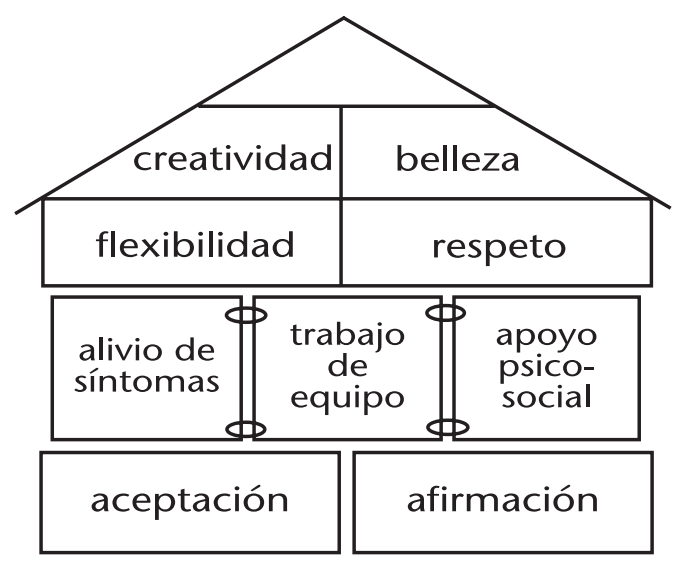

Figura 1. La casa de los hospicios

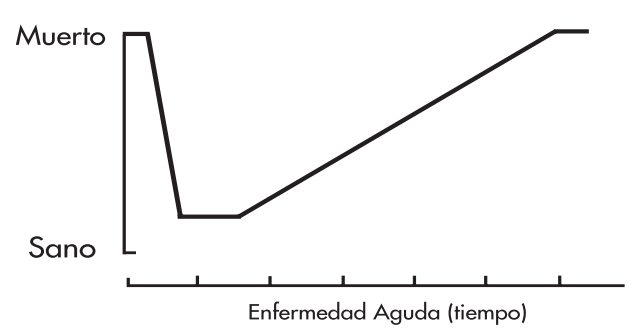

Figura 2. Representación gráfica de enfermedad aguda. Los pronósticos biológicos son generalmente buenos. Las medidas de resucitación aguda son importantes y le permiten al paciente sobrevivir a la crisis inicial. La recuperación se logra con ayuda de las fuerzas naturales de curación, la rehabilitación es completada por el propio paciente, sin la asistencia médica contínua. (Reproducido con autorización de Radcliffe Medical Press).

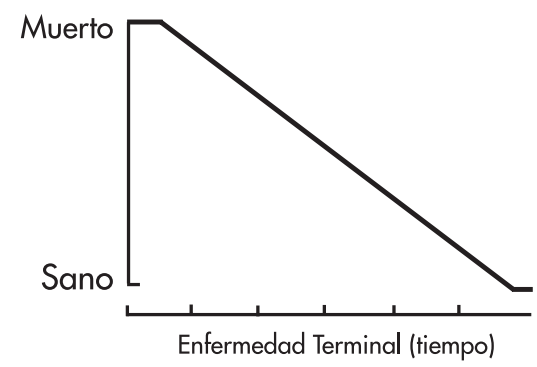

Figura 3. Representación gráfica de enfermedad terminal. Los pronósticos biológicos empeoran progresivamente. Las enfermedades agudas y terminales son, por lo tanto, entidades. patofisiológicas distintas. Las intervenciones terapéuticas, que con propiedad pueden ser descritas como prolongación de la angustia del morir, son tanto futiles como inapropiadas. (Reproducido con autorización de Radcliffe Medical Press).

Tabla 1: Factores que influencian la esperanza en los pacientes terminales

\section{Disminución}

Sentirse devaluado

Abandono y aislamiento:

"conspiración del silencio"

"no hay nada más que hacer"

Falta de orientación/ metas

Dolor y disconfort no controlados

\section{Aumento}

Sentirse valorado

Relaciones significativas:

recuerdos

humor

Metas realistas

Alivio del dolor y síntomas 


\section{Tabla 2: Necesidades de los moribundos}

\section{Dimensión}

Física

Psicológica

Seguridad

Comprensión

Autoestima

Social

Aceptación

Pertenencia

Desprendimiento

Espiritual

Amor

Reconciliación

Autovaloración

Sentido

\section{Necesidad}

Alivio de los síntomas

Sentimiento de seguridad

Explicación de los síntomas y la enfermedad: oportunidad para conversar sobre el proceso de morir Participación en la toma de decisiones, particularmente cuando aumenta la dependencia física de otros; oportunidades para dar y recibir

Actitudes no condenatorias de los profesionales, independientemente de su ánimo, sociabilidad y apariencia

Sentirse necesitado y conectado; no ser una carga

Oportunidad para despedirse de aquellas personas o cosas de las que se está apegado; amarrar los "cabos sueltos" en materia de familia y negocios; traspasar la responsabilidad a otros

Expresiones de afecto; contacto humano, por ejemplo, tacto Oportunidad de sanar las relaciones heridas y de buscar el perdón

Conocimiento de que uno es querido y valorado

Sentimiento de que la propia vida aún tiene un sentido y orientación 


\section{Bibliografía}

Armstrong-Dailey A, Goltzer S Z (eds). Hospice care for children. Oxford: Oxford University Press; 1993.

Cousins A. Anatomy of an illness. New York: Norton; 1979.

Doyle D, Hanks G W, MacDonald N (eds). Oxford textbook of palliative medicine. 2nd ed. Oxford: Oxford University Press; 1997.

Du Boulay S. Saunders: the founder of the modern hospice movement. London: Hodder y Stoughton; 1994.

Infeld D L, Gordon A K, Harper B C (eds). Hospice care and cultural diversity. New York: Haworth Press; 1995.

James N, Field D. The routinization of hospice: charisma and bureaucratization. Social Science Medicine 1992; 34: 1363-1375.

Keown J (ed). Euthanasia examined: ethical, clinical and legal perspectives. Cambridge: Cambridge University Press; 1995.

Kübler-Ross E. On death and dying. London: Tavistock Publications; 1970.

Lewis M. Tears and smiles: the hospice handbook. London: Michael O'Mara Books; 1989.

Mann R D (ed). The history of the management of pain from early principles to present practice. London: Panthenon; 1988.
Mor V. Hospice care systems: structure, process, costs and outcome. New York: Springer Publishing Co.; 1987.

Mount B M. Hospice care. Journal of the Royal Society of Medicine 1980; 73: 471-473.

Organización Mundial de la Salud. Alivio del dolor y tratamiento paliativo en cáncer. Informe de un Comité de expertos. Ginebra: OMS; 1990. (Serie de Informes Técnicos 804).

Sims R, Moss VA. Palliative care for people with AIDS. 2nd ed.. London: Edward Arnold; 1995.

Stedeford A. Facing death: patients, families and professionals. 2nd ed. Oxford: Sobell Publications; 1994.

Stoddard S. Hospice in the United States: an overview. Journal of Palliative Care 1989; 5: 10-19.

Stoddard S. The hospice movement: updated and expanded. A better way of caring for the dying. New York: Vintage Books; 1992.

Working Party Report. Mud and stars: the impact of hospice experience on the Church's ministry of healing. Oxford: Sobell Publications; 1991

World Health Organization. Cancer pain relief and palliative care report. Geneva: WHO; 1990. (Technical Series 804).

Worswick J. A house called Helen. London: Harper Collins; 1993. 